\title{
Verification Experiments of Sail Control Effects on Tracking Oil Spill
}

\author{
Hidetaka Senga, Naomi Kato, Lubin Yu \\ Dept. of Naval Architecture and Ocean Engineering \\ Osaka University \\ Suita, Japan \\ senga@naoe.eng.osaka-u.ac.jp
}

\author{
Muneo Yoshie, Toshinari Tanaka \\ New Technology Development Field \\ Port and Airport Research Institute \\ Yokosuka, Japan \\ yoshie@pari.go.jp
}

\begin{abstract}
It is important to forecast the location of oil spill to realize effective and adequate oil spill response operation when huge oil spill occurs. In order to enhance the accuracy of oil drifting simulation, it is needed to obtain the meteorological and oceanographic data around the oil slick. SOTAB-II is developed for autonomous tracking of oil slick drifting on sea surface. In addition, it transmits its location, the meteorological and oceanographic data around it to the land base in real time. In general, the drifting velocity vector of oil spill contains wind velocity vector and water current velocity vector. SOTAB-II is equipped with a sail whose size and direction are controllable. This paper reports the development of a new SOTAB-II and the results of field experiments using it.
\end{abstract}

Keywords-oil spill; autonomous tracking buoy; sail control; field experiment

\section{INTRODUCTION}

In recent years, there have been many major sea oil spills. The gas and oil blowout accident from seabed also unfortunately happen. These gas and oil damage not only the ocean environment, but also regional economies.

There exist some methods to deal with the oil spill. Drifting buoys are used to track the oil spill [1]. If the sea condition is fine and safe enough, the vessels can track oil spill with using the method of the X-band radar detection [2]. The weak point of these drifting buoys is that when they are once apart from the oil slick, they have no device to track it again. A plane can fly over the sea and detect the oil slick. However, in the night, it is difficult to detect the spilled oil from the plane. Even though aircraft can apply fluorescence lidar system [3] to detect the oil slick in the night, the limitation of its endurance can make it impossible to track the oil slick continuously. Satellite remote sensing is available to detect the location of oil spill [4]. However this method is not carried out more frequently than the air craft.

To overcome the weak points of existing oil spill monitoring methods, we are constructing a system to observe the oil and gas accident. This system consists of the Spilled Oil Tracking Autonomous Buoy (SOTAB) and the land base. Figure 1 shows the concept of this system.

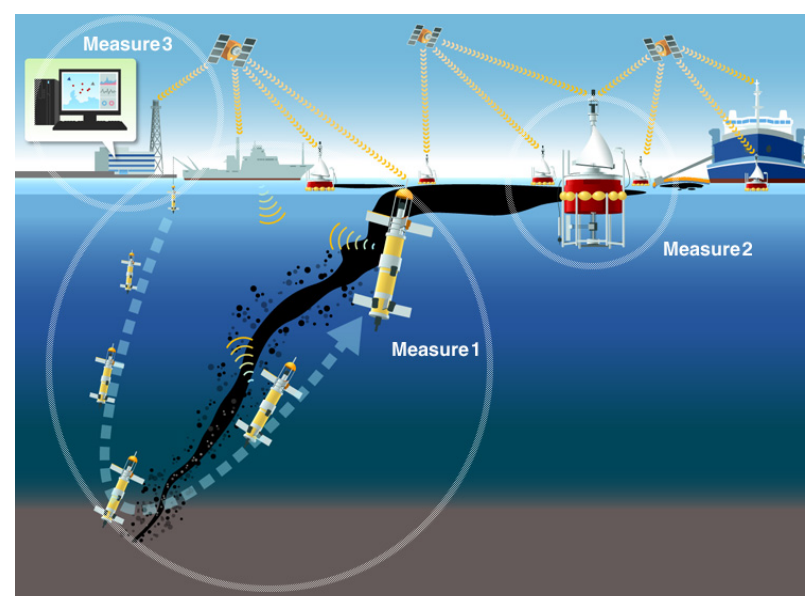

Figure 1. Concept of spilled oil and gas tracking autonomou buoy system.

The underwater autonomous robot (SOTAB-I) observes three dimensional distributions of gas and oil blowing out from seabed (Measure 1). At the sea surface, the autonomous buoy with controllable sail (SOTAB-II) observes the oil slick (Measure 2). Gas and oil drifting simulation and coordination of the oil recovery operations are carried out at the land base (Measure 3). Among these measures, this paper shows the progress of SOTAB-II.

One of the important roles of SOTAB-II is to obtain and send the useful real-time data to the land base. Once the oil blown out from seabed or oil spilled from ship washes ashore, it is difficult to recover it effectively. Such oil must be recovered while it is still drifting on the sea surface. If we can know the real-time data such as the exact location of the drifting oil, the meteorological and oceanographic data around it immediately after the oil spill accident, then oil spill response can be smoothly coordinated. Besides, adequate measures will be taken at coastal areas using information obtained from the oil drifting simulation. This forecast of oil spill is enhanced by data assimilation using the real-time data from SOTAB-II.

This paper reports the development of the new SOTAB-II and some results of the field experiments. 


\section{SOTAB-II}

\section{A. The Configuration of New SOTAB-II}

In general, it is said that the drifting velocity vector of oil spill contains $3.0 \%$ of wind velocity vector along the direction of wind at $10 \mathrm{~m}$ height from sea surface and water current velocity vector. SOTAB-II is equipped with a sail whose size and direction is controllable. An oscillating fin and fixed rudder are installed on it for an auxiliary propulsion mechanism. So as to rapidly respond to the change in the drifting direction of the oil slick, a cylindrical shape is adopted for its hull. A GPS receiver, an anemometer, and current meter are installed on top side of SOTAB-II. After being dropped into oil slick, SOTABII drifts with it by controlling the sail size and direction autonomously. In this drifting mode, SOTAB-II transmits its location, the meteorological and oceanographic data around it to the land base in real-time. Meanwhile, if SOTAB-II became separated from the oil spill or drift away from the main oil slick, an auxiliary propulsion mechanism was used to move into the mail oil slick or the location requested from the land base.

We had developed a prototype of SOTAB-II, and some field experiments were carried out using it in 2010 [5]. However, because of the trouble of the sail control mechanism, only free drifting experiments under different sail size were carried out at that time. The results of free drifting experiments revealed that the wind velocity component of the drifting velocity of SOTAB-II was $3.1 \%$ to $3.8 \%$ of the wind velocity along the wind direction. In case the sail was completely furled, the difference between the drifting direction of SOTAB-II and that of the simulated oil gradually increased as time passed. That difference was decrease in case of completely unfurled sail. These results show the efficiency of the sail for controlling the drifting velocity and direction.

Figure 3 shows the new SOTAB-II developed to evaluate the sail control effects on tracking oil spill. The mass of SOTAB-II is $60 \mathrm{~kg}$. The sail size and sail direction play an important role in gaining wind force. They are controlled by different motors. A roller mounted at the bottom of sail adjusts sail size. By rolling up or down, the sail can be unfurled or furled. Oil detecting sensor is of assistance to figure out if there exist oil slick around SOTAB-II. That sensor is now under development.
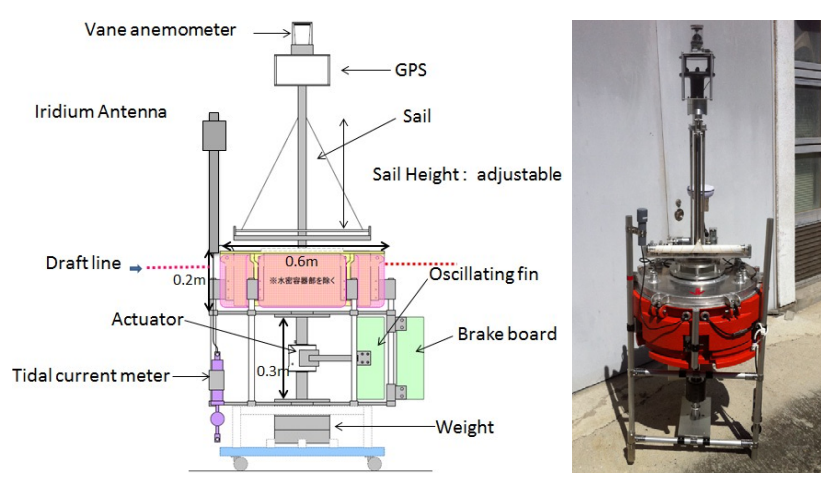

Figure 2. Layout plane (left) and picture of the new SOTAB-II (right).

\section{B. The Control Law of Sail Size and Direction}

The basic law of sail control is as follows. In order to make effectively use of wind force, the sail direction is required to keep perpendicular to the wind direction. On the other hand, if the drifting speed of SOTAB-II is slower than oil slick, the sail size must be increased so as to absorb more wind effect. In case that SOTAB-II drifts faster than oil slick, the sail size must be decreased. The controls of the sail size and sail direction are both conducted by PID controller. Figure 3 show the diagram of these PID control system.
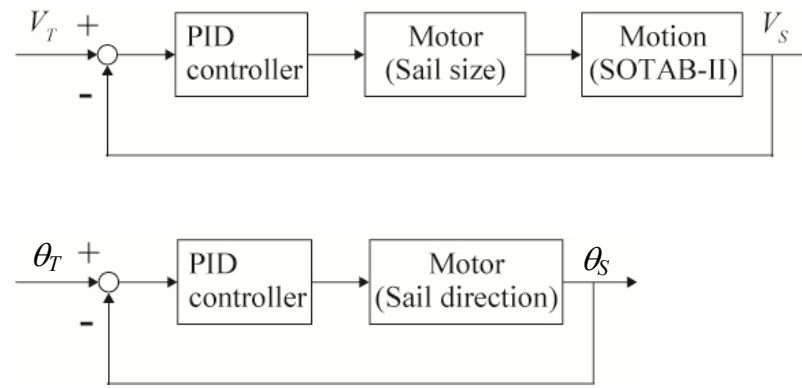

Figure 3. Diagram of PID controller for sail size and sail direction.

In this diagram, $V_{T}$ and $\theta_{T}$ are target drifting velocity in wind direction and target sail angle respectively. $V_{S}$ and $\theta_{S}$ are the drifting velocity of SOTAB-II in wind direction and sail angle of SOTAB-II respectively. Two target values, $V_{T}$ and $\theta_{T}$, are calculated as follows.

SOTAB-II can obtain drifting vector $\vec{V}$ and its direction $\alpha$ from the GPS receiver, where the absolute value of $\vec{V}$ is $V_{S}$. SOTAB-II can also obtain relative wind velocity vector $\overrightarrow{W r}$ and its direction $\gamma$ in the body fixed coordinate system, relative water current velocity vector $\overrightarrow{\mathrm{Cr}}$ and its direction $\zeta$ in the body fixed coordinate system, and azimuth angle $\theta$ by using equipped devices. Figure 4 shows the relations among these values, the body fixed coordinate system $O-X^{\prime} Y^{\prime}$ and earth coordinate system $O-X Y$.

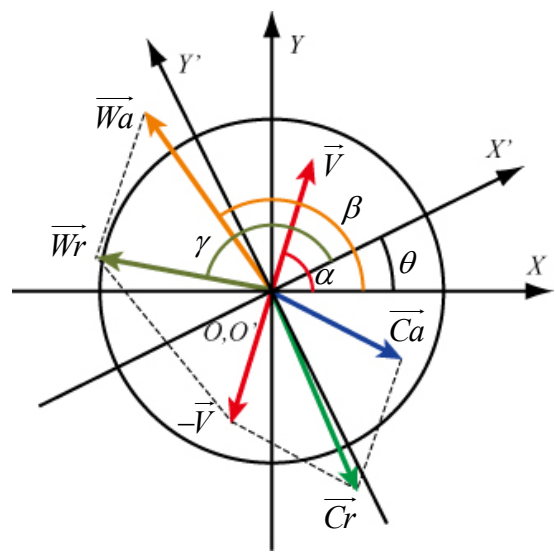

Figure 4. Coordinate system. 
In Fig. $4, \overrightarrow{W a}, \beta, \overrightarrow{C a}$, and $\varepsilon$ represent the absolute wind velocity vector, its direction, absolute water current velocity vector, and its direction in the earth coordinate system respectively. These values are calculated by following relations.

$$
\begin{aligned}
& \overrightarrow{W a}=(\text { Wax }, \text { Way }) \\
& =(|\overrightarrow{W r}| \cos (\gamma+\theta)+|\vec{V}| \cos \alpha,|\overrightarrow{W r}| \sin (\gamma+\theta)+|\vec{V}| \sin \alpha) \\
& \beta=\tan ^{-1}(\text { Way } / \text { Wax }) \\
& \overrightarrow{C a}=(\text { Cax }, \text { Cay }) \\
& =(|\overrightarrow{C r}| \cos (\varsigma+\theta)+|\vec{V}| \cos \alpha,|\overrightarrow{C r}| \sin (\varsigma+\theta)+|\vec{V}| \sin \alpha) \\
& \varepsilon=\tan ^{-1}(\text { Cay } / \text { Cax })
\end{aligned}
$$

As is well known, the drifting velocity vector of oil spill contains wind effect and water current effect. We assume that the water current component of the drifting velocity of oil slick and SOTAB-II are same. The wind component of the drifting velocity of SOTAB-II $V_{W}$ is calculated by eq. (5).

$$
V_{W}=|V S| \cos (\beta-\alpha)-|\overrightarrow{C a}| \cos (\beta-\varepsilon)
$$

Generally, the wind component of the drifting velocity of oil slick is approximately $3.0 \%$ of wind velocity along the direction of wind at $10 \mathrm{~m}$ height from sea surface. The height from sea surface is $0.4 \mathrm{~m}$ that the anemometer installed in SOTAB-II. Thus, $\overrightarrow{W r}$ and $\overrightarrow{W a}$ are wind data at $0.4 \mathrm{~m}$ height from the sea surface. These data can be transformed into those at a height of $10 \mathrm{~m}$ from sea surface by using following equations [6].

$$
\begin{gathered}
U_{(z)}=\frac{u^{*}}{\kappa} \log \frac{z}{z_{0}} \\
z_{0}=0.0185\left(u^{*}\right)^{2} / g \\
u^{*}=\sqrt{\tau_{s} / \rho_{a}} \\
\tau_{s}=\rho_{a} C_{f} U_{(10)}^{2}
\end{gathered}
$$

where,

$U_{(z)} \quad$ : wind velocity at a $\mathrm{z}[\mathrm{m}]$ height from the sea surface $\mathrm{z} \quad$ : height from the sea surface

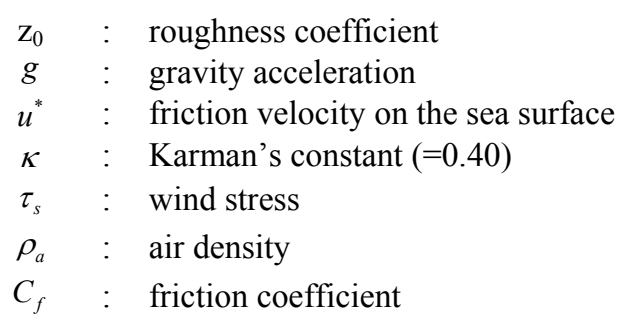

From these equations and absolute wind velocity at $0.4 \mathrm{~m}$ height from the sea surface $\overrightarrow{W a}$, we can obtain the wind velocity at $10 \mathrm{~m}$ height from sea surface $U_{(10)}$. In this research, we assume that the wind velocity component of oil slick drifting is $3.0 \%$ of $U_{(10)}$. $U_{(10)}$ and the target drifting velocity in wind direction $V_{T}$ for sail size control are as follows.

$$
U_{(10)}=\frac{\kappa|\overrightarrow{W a}|}{\sqrt{C_{f}} \log \frac{z}{z_{0}}}
$$

$$
V_{T}=0.03 U_{(10)}
$$

Meanwhile, the sail direction is controlled to be perpendicular to the absolute wind direction at any times to fully utilize the wind effect. The target sail angle $\theta_{T}$ for sail direction control is expressed by the following equation.

$$
\theta_{T}=\beta-\theta+90
$$

\section{EXPERIMENTS AT LAKE}

\section{A. Purpose of Experiments and Experimental Conditions}

The field experiments in lake were carried out mainly aimed at confirming the validity of the control systems. They also included that the comparisons of the drifting characteristics between free drifting condition without the sail control and the sail controlled condition. These experiments were carried out on Sep. $29 \sim$ Sep.31 in Biwa lake that is the largest lake in Japan (Fig. 5). The average wind velocity ranged from $3.5 \mathrm{~m} / \mathrm{s}$ to $6.3 \mathrm{~m} / \mathrm{s}$, and the current velocity varied from $0.08 \mathrm{~m} / \mathrm{s}$ to $0.19 \mathrm{~m} / \mathrm{s}$.

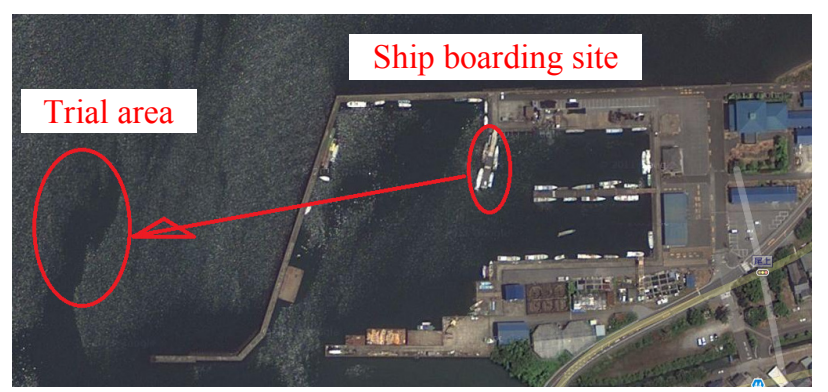

Figure 5. Experimental site (Biwa lake) 
It was reported that the use of thin floating objects such as the rubber sheet was efficient for simulating the drift of oil slick [7]. In these experiments, the rubber sheet was used as a maker for visual confirmation.

After getting out of the port by ship, SOTAB-II and a rubber sheet were dropped into the water at the same time and roughly in same position. Three kinds of sail control condition were investigated. First condition was free drift with sail furled. Second one was free drift with sail completely unfurled. In third condition, the sail size and direction were controlled by PID controller. Figure 6 shows the overview of these experiments in lake.

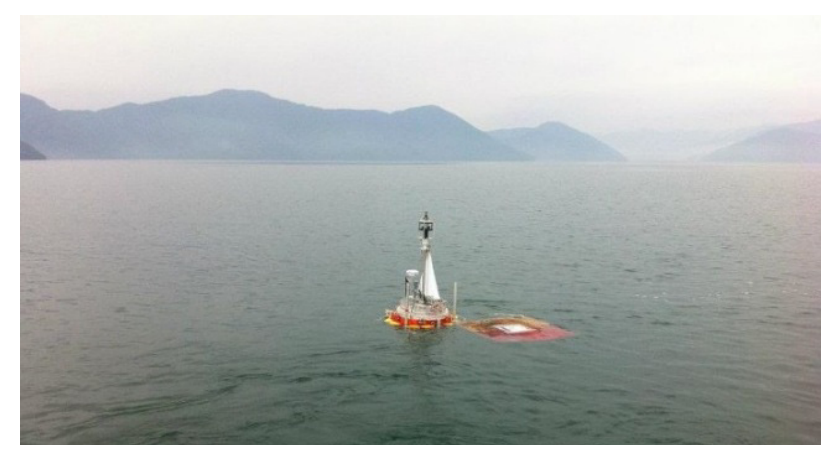

Figure 6. Overview of the experiment at Biwa lake

\section{B. Experimental Results of Biwa lake}

1) Sail direction control: The sail direction is required to be perpendicular to the absolute wind direction all the time. Figure 7 shows the results of sail direction relativee to wind direction under the condition of free drift and sail controlled. The sail direction was in a random angle relative to wind direction under the condition of free drift. On the contrary, we can confirm that the sail direction always kept perpendicular to sail direction under the condition of sail control.

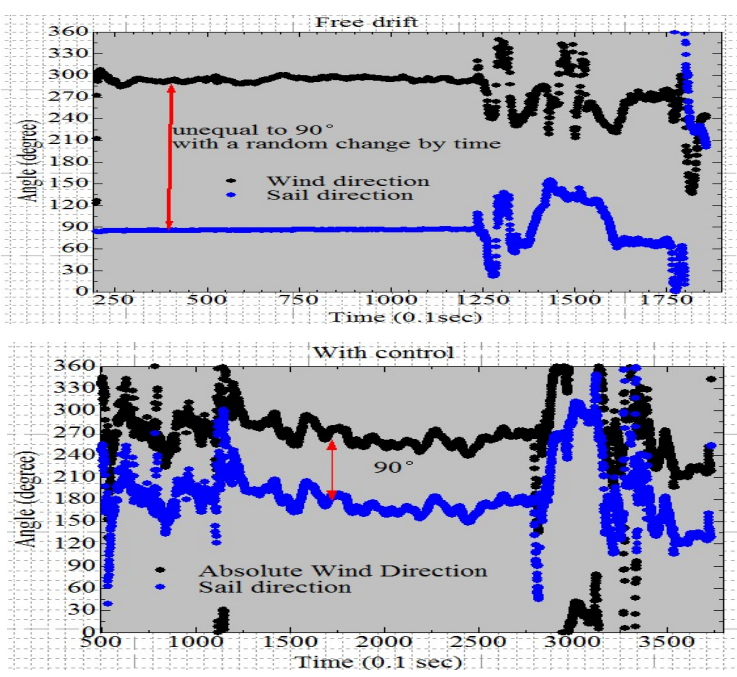

Figure 7. Sail direction relative to absolute wind direction
2) Effectiveness of sail and sail control: It was expected that when SOTAB-II drifts faster than the target value $V_{T}$, the sail will furl to decrease the effect of wind. On the other hand, when SOTAB-II drifts slower than $V_{T}$, the sail size will be increased to obtain more wind force. Figures 8show the component of SOTAB-II's drifting velocity in wind direction $V_{S}$ under the different sail conditions. The water current velocity in the wind direction was excluded from the drifting velocity of SOTAB-II in wind direction. Under the condition of sail control, the average drifting velocity of SOTAB-II in wind direction is approximately $70.8 \%$ of the average of $V_{T}$. On the other hands, under the condition of free drift with sail unfuled and fuled, those persentages decrease to $56.9 \%$ and $43.29 \%$ respectively. Through the whole experiment, $V_{S}$ was lower than $V_{T}$. These decreses in $V_{S}$ are mainly caused by the additional floats attached on the side of SOTAB-II's hull. Even though the velocity of SOTAB-II cannot reach the target drifting velocity in wind direction $V_{T}$ and the drag force on SOTAB-II should be decreased, these results show the efficiency of the sail and sail control.
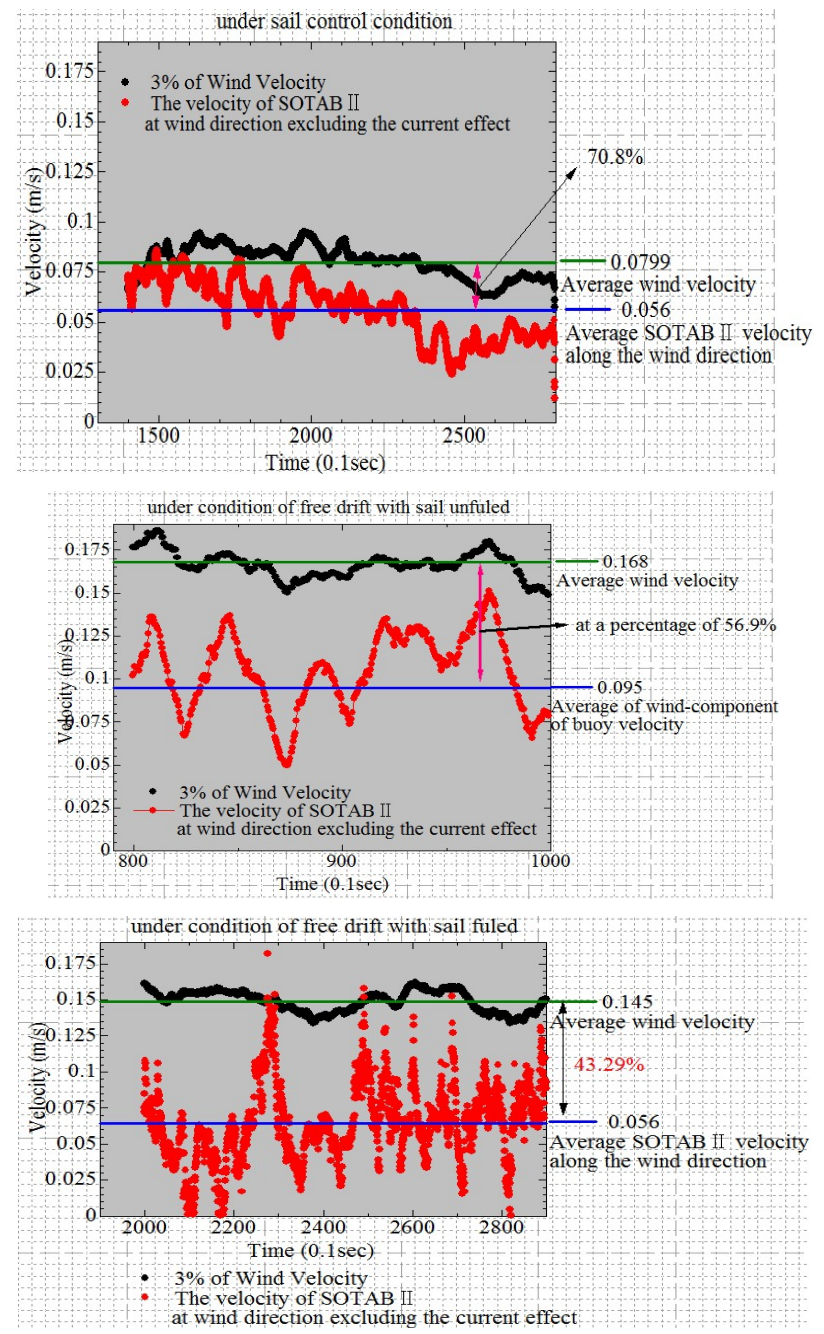

Figure 8. Comprrison $V_{S}$ and $V_{T}$ under different sail condition. 
3) Trajectories of SOTAB-II: The trajectories of SOTABII were calculated from the GPS data. Basec on the assumption in this study that the wind component of the drifting vector of oil splick was $3 \%$ of the wind vector at a height of $10 \mathrm{~m}$ above the sea surface, the trajectories of imaginary oil slick were simulated using above estimated the wind velocity component of oil slick drifting is $3.0 \%$ of $U_{(10)}$ and absolute water current $\overrightarrow{\mathrm{Ca}}$. Figures 9 show these trajectories comparisons under the condition of free drift with sail completely furled and sail control. In these figures, black line shows the trajectory of SOTAB-II. The yellow and red line show the trajectory of imaginary oil slick calculated by the effect of only the water current and $3.0 \%$ of $U_{(10)}$ respectively. The green line shows the trajectory that calculated by the summention of both effects. The circles on each trajectory indicate the location of each item every $60 \mathrm{sec}$. We can confirm that the trajectories of SOTAB-II (black line) almost lie midway between the yellow and red line. The total length of each line indicates the drifting distance at each experiment. The difference in length between the black and green line under sail control condition is smaller than that of free drift with sail completely furled. Besides, the gap between black and green line under sail control condition is smaller than that of free drift with sail completely furled. This result also shows the efficiency of sail and sail control.
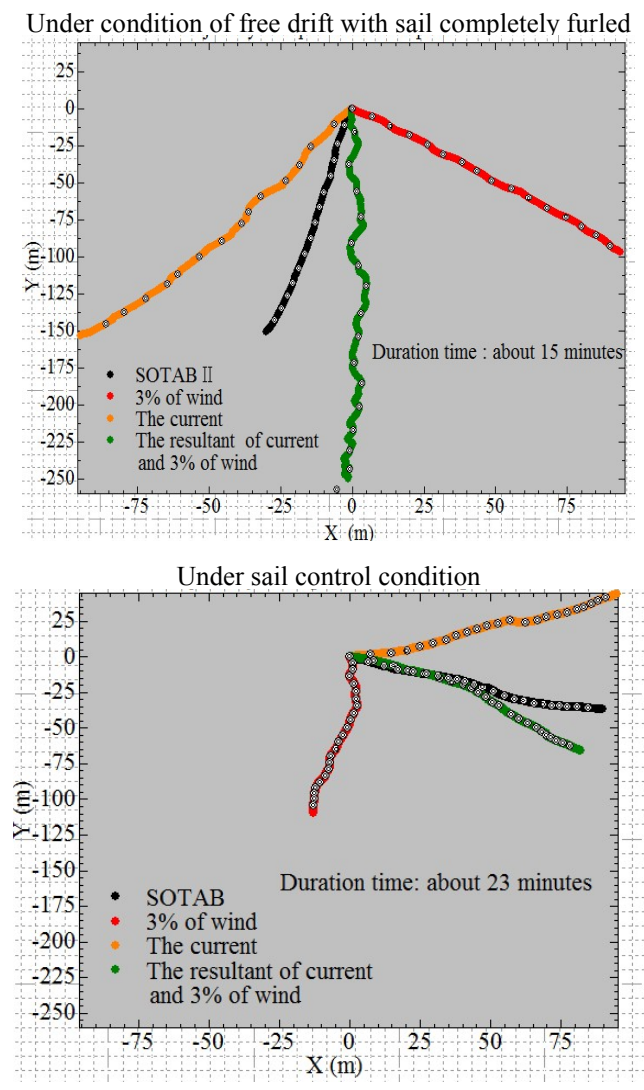

Figure 9. Trajectories of SOTAB-II and that of simulated oil slick.

\section{EXPERIMENTS AT SEA}

\section{A. Purpose of Experiments and Experimental Conditions}

The experimental results at lake strongly demonstrate the effectiveness of the sail and sail control. SOTAB-II with sail controlled was able to approach to the direction of simulated oil spill. However, SOTAB-II was unable to reach the target drifting velocity $V_{T}$. The auxiliary propulsion mechanism such as oscillating fin and fixed rudder were removed from SOTABII to decrease the drag force on SOTAB-II in water. Drifting experiments at sea were carried out using this SOTAB-II.

Experimental site was Osaka Bay about $7 \mathrm{~km}$ away from Awaji Island in Japan. Experimental period is from Dec. 21th to Dec. 23th. In this period, the average wind velocity ranged from approximately $8.8 \mathrm{~m} / \mathrm{s}$ to $11.5 \mathrm{~m} / \mathrm{s}$, and the water current velocity varied from $0.34 \mathrm{~m} / \mathrm{s}$ to $0.59 \mathrm{~m} / \mathrm{s}$, which were $2 \sim 3$ times as large as those in experiments at Biwa lake.

Same as previous experiment in lake, a rubber sheet was first released into the sea, and then SOTAB-II was dropped (Fig. 10). That rubber sheet was equipped with a GPS receiver so that the velocity and trajectory of the imaginary oil slick can be calculated. Three kinds of sail control condition, free drift with sail furled, free drift with sail completely unfurled, and sail controlled were investigated. In this time, same as the experiment at Biwa lake, the target drifting velocity in wind direction $V_{T}$ for sail control was set as $3.0 \%$ of $U_{(10)}$.

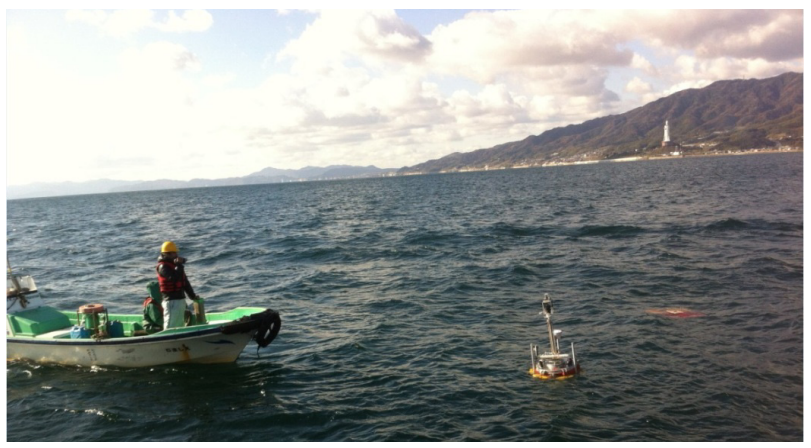

Figure 10. Overview of the experiment at sea

\section{B. Experimental Results of Sea}

1) Drifting velocity of rubber sheet: In the same manner as experiments at lake, the drifting velocity of rubber sheet in wind direction was calculated. The water current velocity in the wind direction was excluded from it. As a result, its average was approximately from $1.8 \%$ to $1.95 \%$ of the wind velocity at the $10 \mathrm{~m}$ height above the sea surface. This means it reached approximately $60 \% \sim 65 \%$ of $V_{T}$. Figure 11 shows one example of the rubber sheet's drifting velocity in wind direction. That velocity of SOTAB-II is also plotted in this figure. In comparison with the drifting velocity of SOTAB-II under the sail completely unfrled condition, the drifting velocity of rubber sheet in wind direction was close to that of SOTAB-II. From the other results, it was smaller and larger than that of SOTAB-II under the sail completely unfruled and furled condition respectively. 


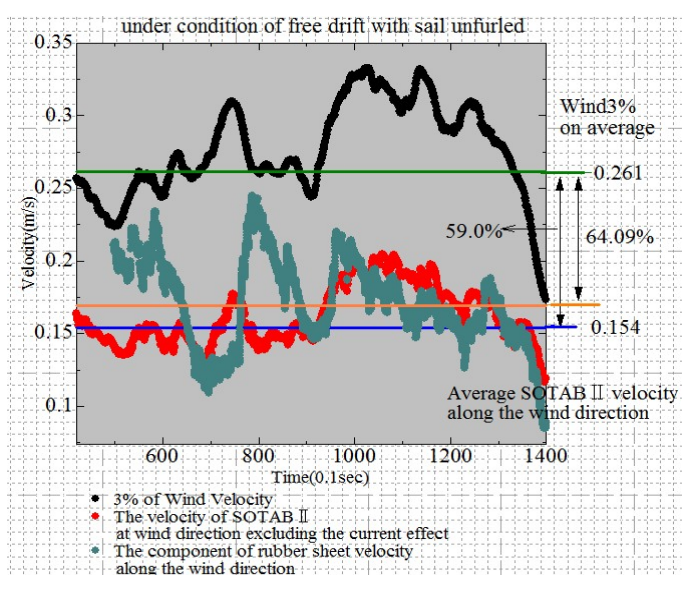

Figure 11. The drifting velocity of the rubber sheed in wind direction.

As shown in Fig. 11, even though the auxiliary propulsion mechanism had been removed, the drifting velocity of SOTABII in wind direction couldn't reach the target value " $3.0 \%$ of $U_{(10)}$ ". This target value is determined according to the general assumption. We should reconsider the percentage of the wind effect because it is not deterministic value and varies according to the sea conditions. However, if a sensor to detect the oil slick near itself is installed on SOTAB-II, it is easy to correct automatically that target value on site.

2) Trajectories of SOTAB-II and rubber sheet: The trajectories of SOTAB-II and rubber sheet were calculated from the GPS data. The trajectories of imaginary oil slick were also calculated in the same manner as the analysis of experiment at Biwa lake. Figures 12 show these trajectories comparisons under the condition of free drift with sail completely furled, completerly unfurled, controlled. In these figures, black line shows the trajectory of SOTAB-II. The yellow and red line show the trajectory of imaginary oil slick calculated by the effect of only the water current and $3.0 \%$ of $U_{(10)}$ respectively. The green line shows the trajectory that calculated by the summention of both effects. The blue line indicates the trajectory of rubber sheet. The circles on each trajectory indicate the location of each item every $60 \mathrm{sec}$.

The trajectory of SOTAB-II and that of rubber sheet are always lie midway between the yellow and red line. Besides, in all cases, the black ang blue line lie between yellow and green line. This indicates that the percentage of the wind effects on SOTAB-II and rubber sheet were smaller than 3\%.

The lenght of each trajectory indicates the drifting distance during each experiment. The difference in length between black and green line under sail control condition is smaller than that of under the condition of free drift with sail completely furled. Meanwhile, by comparing the drifting distance of SOTAB-II and that of rubber sheet, only the SOTAB-II under sail control condition reached farther than rubber sheet, and it was closest to the end of the green line. This means not only the effectiveness of the sail but also the effectiveness of the sail control. It is supposed that SOTAB-II can drift with the oil slick if its drag force in water is reduced so that its drifting velocity in wind direction becomes $3.0 \%$ of $U_{(10)}$. However, as mentioned before, the target value " $3.0 \%$ of $U_{(10)}$ " is determined according to the general assumption. This percentage of wind effect should be corrected according to the sea conditions.
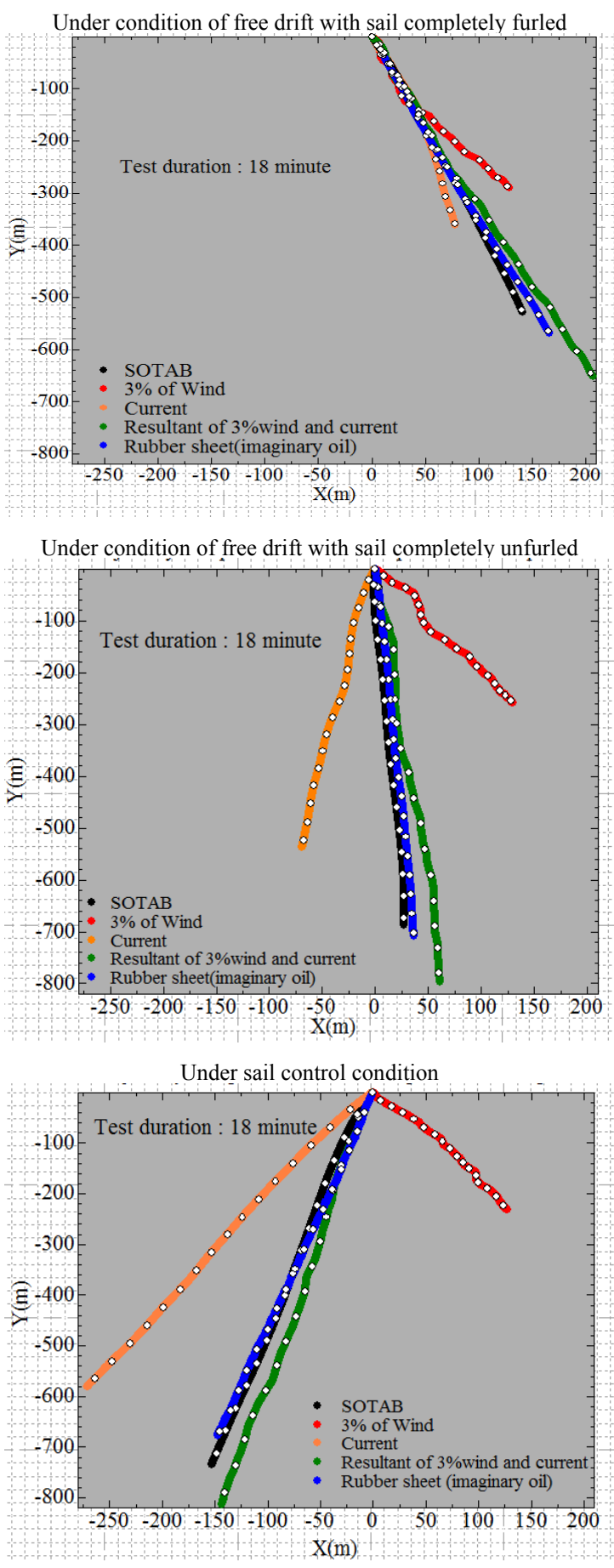

Figure 12. Trajectories of SOTAB-II, rubber sheet, and simulated oil slick. 


\section{CONCLUSIONS}

We are constructing a system to observe the oil and gas accident in the ocean. In this system, the autonomous buoy named SOTAB-II observes and tracks the oil slick drifting on the sea surface. The drift of oil slick on the sea is affected not only the water current but also the wind. SOTAB-II has a sail whose size and direction are controllable. After it is dropped into the oil slick drifting on the sea surface, SOTAB-II sets out to drift with the oil slick by controlling the sail size and direction to effectively gain the wind force.

In this research, we carried out some experiments at lake and sea with using SOTAB-II. A rubber sheet with a GPS receiver was also dropped into the sea for visual confirmation and obtaining the drifting data of floating on the sea surface. The trajectory of imaginary oil slick was also simulated with using the water current velocity and the wind velocity measured by SOTAB-II. In these field experiments, the sail control system performed correctly. The sail direction is controlled to be perpendicular to the absolute wind direction at any time. On the other hands, the sail size is controlled so that the drifting velocity of SOTAB-II in wind direction becomes $3.0 \%$ of $U_{(10)}$. Experimental results and its analysis show the efficiency of the sail and sail control. The sail system installed on SOTAB-II brings a great similarity of the drifting velocity, direction, trajectory between SOTAB-II and imaginary oil slick Even though the basic drifting performance and tracking oil spill function have been confirmed, it is needed to investigate the exact proportion of oil spill velocity to the wind velocity, and develop the oil detecting sensor for SOTAB-II.

\section{ACKNOWLEDGMENT}

This research project is being funded for 2011FY-2015FY by Grant-in-Aid for (Scientific Research(S) of Japan Society for the Promotion of Science(No. 23226017).

\section{REFERENCES}

[1] R.H. Goodman, D. Simecek-Beatty, and D. Hodginse, "Tracking Buoys for Oil Spills,” Proc. Of Int. Oil Spill Conf., API, 1999, pp.3-8.

[2] MIROS, Plc, http://www.miros.no/doc/oil_spill_report_2005.pdf.

[3] S. Yamagishi, K. Hitomi, H. Yamanouchi, Y. Yamaguchi and T. Shibata, "Development and Test of a Compact Lidar for Detection of Oil Spills in Water," Proc. PIIE 4154, 2000, pp.136-144.

[4] H.V. Jensen, J.H.S. Andersen, P.S. Daling and E. Nost, "Recent Experience from Multiple Remote Sensing and Monitoring to Improvement Oil Spill Response Operations,” 2008 Int. Oil Spill Conf., 2008, pp.407-412.

[5] H. Senga, N. Kato, H. Suzuki, M. Yoshie, I. Fujita, T. Tanaka and Y. Matsuzaki, "Development of a New Spilled Oil Tracking Autonomous Buoy," Marine Technology Society Journal, 2011, Vol.45, Number 2, pp.43-51.

[6] U. Sanae, Physical Oceanography of the Coast, Japan, Tokai University Press, 1993.

[7] Y. Matsuzaki, M. Yoshie, I. Fujita and K. Takezaki, "Drift Experiment using Thin Floating Mat and Study to Predict Drift," Annual Journal of Civil Engineering in the Ocean, 2009, Vol.25, pp.33-38 